\title{
Vasopressin withdrawal associated with massive polyuria
}

\author{
Candy S. Peskey, PharmD, ${ }^{\mathrm{a}}$ William J. Mauermann, $\mathrm{MD},{ }^{\mathrm{b}}$ Steven R. Meyer, $\mathrm{MD}, \mathrm{PhD},{ }^{\mathrm{c}}$ and \\ Martin D. Abel, MD, ${ }^{\mathrm{d}}$ Rochester, Minn
}

Vasopressin has demonstrated success in the treatment of vasodilatory shock. ${ }^{1}$ It is also frequently used to manage decreased systemic vascular resistance (SVR) during or after cardiac surgery with cardiopulmonary bypass. We report a case of massive polyuria and increasing serum tonicity after withdrawal of a vasopressin infusion in a patient after cardiac surgery.

\section{CLINICAL SUMMARY}

A 32-year-old man with Marfan's syndrome treated with atenolol and lisinopril underwent repair of an ascending aortic aneurysm, closure of a patent foramen ovale, and mitral valve repair. After termination of cardiopulmonary bypass, a vasopressin infusion $(0.08 \mathrm{U} / \mathrm{min})$ was initiated for persistent hypotension caused by decreased SVR (456 dynes . $\mathrm{sec}^{-1} \cdot \mathrm{cm}^{-5}$ ). The vasopressin infusion was continued for 3 days, at which time the infusion was withdrawn over 12 hours. During this period, the patient was awake and alert and had free access to oral fluids.

Within 3 hours of discontinuing the vasopressin infusion, the patient's urine output increased to more than $1 \mathrm{~L} / \mathrm{h}$ without any other medication changes, and oral fluid intake averaged $300 \mathrm{~mL} / \mathrm{h}$ (Figure 1). Specifically, no diuretics had been administered for at least 24 hours. The serum sodium concentration (normal range, $135-145 \mathrm{mmol} / \mathrm{L}$ ) increased to $135 \mathrm{mmol} / \mathrm{L}$ from $129 \mathrm{mmol} / \mathrm{L}$. During the diuresis, urine osmolality measured 164 mOsm (normal, 300-800 mOsm/ $\mathrm{kg}$ ), and serum osmolality was $272 \mathrm{mOsm}$ (normal, 275$295 \mathrm{mOsm} / \mathrm{kg}$ ). The excretion of large volumes of dilute urine with an increasing systemic tonicity is consistent with diabetes insipidus, and we presumed this to be the diagnosis. ${ }^{1}$ On administration of $25 \mu \mathrm{g}$ of desmopressin intravenously, the urine output decreased to an average of $66 \mathrm{~mL} / \mathrm{h}$ over 6 hours, and urine osmolality increased to $623 \mathrm{mOsm} /$ $\mathrm{kg}$, suggesting a diagnosis of central diabetes insipidus. A subsequent dose of desmopressin was required 24 hours later for increased urine output.

\footnotetext{
From the Mayo Clinic Rochester Department of Pharmacy ${ }^{\mathrm{a}}$; the Department of Anesthesiology, Division of Cardiovascular Anesthesiology ; the Department of Cardiac Surgery, Division of Cardiovascular Surgery ${ }^{\mathrm{c}}$; and the Department of Anesthesiology, Division of Cardiovascular Anesthesiology, ${ }^{\mathrm{d}}$ Mayo Clinic Rochester, Rochester, Minn.

Supported by departmental and institutional support.

Received for publication Feb 13, 2008; revisions received May 22, 2008; accepted for publication June 15, 2008; available ahead of print Aug 4, 2008.

Address for reprints: William J. Mauermann, MD, 200 First St SW-Mb 2-752, Rochester, MN 55909 (E-mail: mauermann.william@mayo.edu).

J Thorac Cardiovasc Surg 2009;138:491-2

$0022-5223 / \$ 36.00$

Copyright $(c) 2009$ by The American Association for Thoracic Surgery

doi:10.1016/j.jtcvs.2008.06.032
}

\section{DISCUSSION}

Arginine vasopressin, or antidiuretic hormone, is a nonapeptide hormone produced in the hypothalamus and stored in and released from the posterior pituitary gland. ${ }^{1}$ Vasopressin release occurs primarily in response to increased serum osmolality, as well as hypotension and hypovolemia. Vasopressin activates V1 receptors in blood vessels, causing vasoconstriction; V2 receptors in the collecting tubules of the kidney, promoting water reabsorption; and V3 receptors in the central nervous system, regulating corticotropin-releasing hormone and possibly other neurotransmitters. ${ }^{1}$

Although vasopressin is used in the setting of decreased SVR for its vasoconstrictive properties, the increase in plasma volume caused by free water reabsorption augments its effect on blood pressure. ${ }^{2}$ Adverse effects associated with the use of vasopressin in hypotension are caused by either this vasoconstriction or an increase in free water. Most of these effects are mild and include blanching of the skin, abdominal cramping, and nausea. Rare complications include water intoxication, acute coronary syndrome, and ischemic colitis.

Vasoplegia after cardiac surgery is increasingly being recognized and might be related to the use of angiotensin receptor blockers or angiotensin-converting enzyme inhibitors, which this patient was taking preoperatively. ${ }^{3}$ In this report we describe a case of massive polyuria that occurred in association with the withdrawal of vasopressin to treat vasoplegia after cardiac surgery. There are 3 possible explanations: primary polydipsia, iatrogenic hyponatremia, or transient central diabetes insipidus.

Primary polydipsia is defined as the compulsive ingestion of water, resulting in hyposmolality. In the face of fluid deprivation, these patients are able to concentrate their urine and normalize their serum osmolality. We cannot exclude this explanation for the patient's clinical condition because a fluid deprivation test was not performed. However, an increasing serum sodium level makes this unlikely.

Iatrogenic hyponatremia caused by the use of a vasopressin infusion might have caused a syndrome similar to that seen with inappropriate antidiuretic hormone secretion. The discontinuation of vasopressin in this patient might have led to compensatory diuresis with the production of a large volume of dilute urine that in turn resulted in an increase in serum sodium and osmolality. However, this explanation does not account for the polydipsia seen in this patient after discontinuation of vasopressin. Vasopressin infusions are commonly used to treat vasoplegia after cardiac surgery. To our knowledge, iatrogenic hyponatremia caused by the use of vasopressin in this setting has not been reported. 


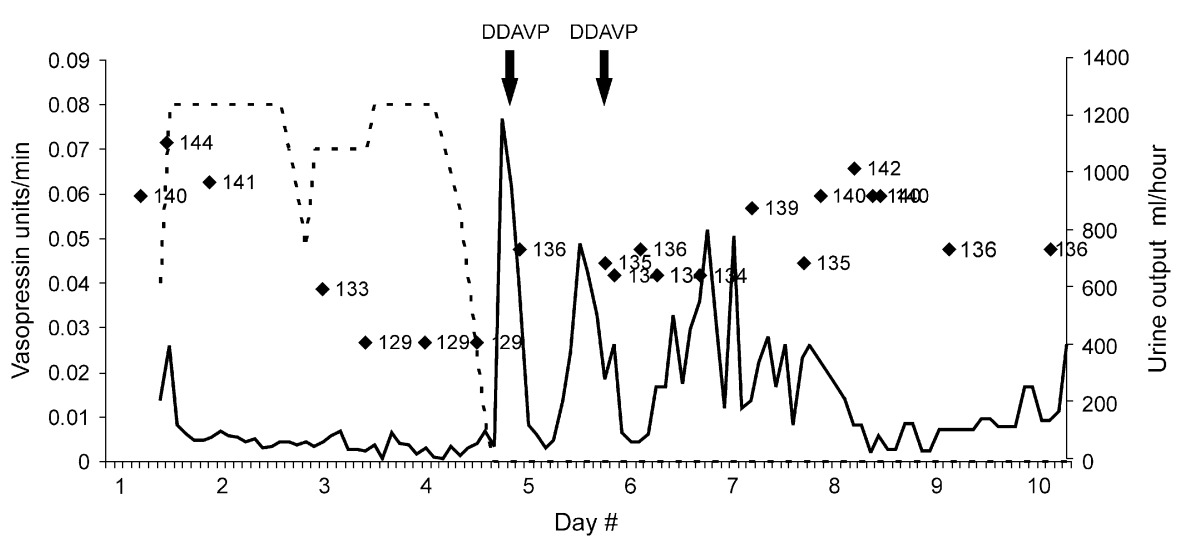

FIGURE 1. Temporal relationship of vasopressin infusion (dashed line, left ordinate), urine output (solid line, right ordinate), and serum sodium concentration (diamonds; in millimoles per liter). DDAVP, (desmopressin).

A third possible explanation for this patient's clinical presentation is a form of transient central diabetes insipidus. Central diabetes insipidus is caused by a deficiency of endogenous vasopressin that results in polyuria and hyperosmolality. In the awake patient the resultant polydipsia maintains normal serum osmolality in the face of the production of large volumes of dilute urine. Fluid deprivation in such a patient would result in hyperosmolality and hypernatremia. In the patient described in this report, we speculate that the withdrawal of vasopressin resulted in a transient form of central diabetes insipidus, causing polyuria and an increasing serum sodium level. Treatment with desmopressin proved effective in normalizing urine output and preventing hypernatremia. We cannot be certain of this diagnosis because a fluid deprivation test was not performed and the patient had ready access to an unlimited supply of fluids. The occurrence of transient diabetes insipidus resulting from the withdrawal of vasopressin infusions theoretically might be due to negative-feedback inhibition of endogenous vasopressin production and release occurring through the V3 receptor. Alternatively, endogenously synthesized vasopressin in this patient might have been depleted because of increased demand from the hypovolemic state, and withdrawal of exogenously administered vasopressin simply unmasked this deficit. ${ }^{4}$

Regardless of the diagnosis or mechanism, polyuria and changes in serum tonicity after withdrawal of exogenously administered vasopressin appear to resolve spontaneously over time. In the case described here, only a short course of vasopressin agonist therapy was required to temporarily maintain free water balance. Overall, there are few reported adverse effects of vasopressin, despite increasing therapeutic use. The occurrence of polyuria and increasing serum sodium after continuous vasopressin administration represents an uncommon but serious possibility and warrants ongoing surveillance.

\section{References}

1. Treschan TA, Peters J. The vasopressin system: physiology and clinical strategies. Anesthesiology. 2006;105:599-612; quiz 39-40.

2. Albright TN, Zimmerman MA, Selzman $\mathrm{CH}$. Vasopressin in the cardiac surgery intensive care unit. Am J Crit Care. 2002;11:326-32.

3. Shanmugam G. Vasoplegic syndrome-the role of methylene blue. Eur J Cardiothorac Surg. 2005;28:705-10.

4. Sharshar T, Carlier R, Blanchard A, et al. Depletion of neurohypophyseal content of vasopressin in septic shock. Crit Care Med. 2002;30:497-500. 